\title{
Neuroprotective and Neurodegenerative Aspects of Coffee and Its Active Ingredients in View of Scientific Literature
}

\author{
Shehnaz Wasim ${ }^{1}$, Vishal Kukkar ${ }^{2}$, Vanessa M. Awad ${ }^{3}$, Sirisha Sakhamuru ${ }^{1}$, Bilal Haider Malik ${ }^{1}$ \\ 1. Internal Medicine, California Institute of Behavioral Neurosciences and Psychology, Fairfield, USA 2. Radiology, \\ California Institute of Behavioral Neurosciences and Psychology, Fairfield, USA 3. Internal Medicine/Family Medicine, \\ California Institute of Behavioral Neurosciences and Psychology, Fairfield, USA
}

Corresponding author: Shehnaz Wasim, shehnaz.wasim@yahoo.com

\begin{abstract}
Coffee and its components have several neuroprotective properties that lower the risk of cognitive decline and other neurodegenerative diseases. This study reviews the mechanisms by which coffee and its respective compounds affect the brain and its pathologies. Many epidemiological studies in this literature review have shown coffee to reduce the risk of developing dementia, stroke, and Alzheimer's disease. It may also have a positive impact on the disease course of amyotrophic lateral sclerosis, Parkinson's disease, and depression. The optimal benefits achieved from coffee in these pathologies rely on higher daily doses. Most of its effects are attributed to caffeine by the antagonism of adenosine receptors in the central nervous system; however, other coffee constituents like chlorogenic acids have also shown much promise in therapeutic value. Existing research considers coffee to have great potential, but additional studies are still needed to clarify the mechanisms and actual causal relationships in certain neuropathologies.
\end{abstract}

Categories: Neurology, Psychiatry, Public Health

Keywords: coffee consumption, caffeine, cognitive decline, alzheimers disease, dementia, stroke, neurodegenerative disesase, parkinson's disease

\section{Introduction And Background}

Coffee is one of the most popular beverages. On average, Americans drink about two cups of coffee everyday [1]. Its exports worldwide amounted to more than 11 million $60 \mathrm{~kg}$ bags in March 2020 alone, posing a significant impact on the health of its consumers on such a population scale; therefore, studies regarding its effects have peaked in recent years [2].

Received 07/17/2020 Review began 07/19/2020 Review ended 07/28/2020 Published 08/05/2020

(c) Copyright 2020

Wasim et al. This is an open access article distributed under the terms of the Creative Commons Attribution License CC-BY 4.0., which permits unrestricted use, distribution, and reproduction in any medium, provided the original author and source are credited.
Coffee contains more than a thousand compounds, many of which have yet to be found [3]. Its bioactive components include the most widely known caffeine and others, like chlorogenic acids (CGAs), diterpenes, trigonelline, tryptophan alkaloids, and secondary metabolites that are a product of Maillard reactions called melanoidins [4]. These have anti-inflammatory, antioxidant, antifibrotic, antimicrobial, and anti-cancer properties that lead to its beneficial role in lowering all-cause mortality and improving endocrine, liver, gastrointestinal, cardiovascular diseases, cancers, and, to which our paper focuses on, neurocognitive function and neurodegenerative diseases [5-7].

Coffee undergoes a number of chemical reactions starting from the type of bean (Arabica or Robusta) used to the level of roasting and the method of preparation by brewing or grind setting [8-10]. This altogether will determine the final composition of the cup of coffee, and the bioavailability of ingredients will depend on each individual's metabolism [11].

Moderate coffee intake (e.g., two to four cups, which is $200-400 \mathrm{mg}$ ) in a day is associated with the highest benefits, while risks are relatively low [12].

This popular beverage has reduced the risk of numerous neurological disorders such as Parkinson's disease (PD), depression, and neurocognitive decline, especially seen in Alzheimer's disease (AD) [5]. The reason for its popularity is the short-term effects that include raising alertness and energy majorly due to caffeine inhibiting adenosine receptors in the brain; however, higher doses (400-800 $\mathrm{mg}$ at one time) can account for anxiety, jitteriness, insomnia, and tachycardia [13]. Apart from caffeine, there are many more effects on the brain associated with other potential therapeutic components of coffee [14].

Observational studies provide evidence of coffee having an association with a myriad of benefits over different diseases. Still, very few have shown causal relations especially in a dose-dependent manner; therefore, more long-term randomized controlled trials will be needed with effective methods to eliminate confounding factors such as smoking to understand the actual therapeutic value of not only coffee as a whole but also its separate components, each having specific effects of their own. This study summarizes the 


\section{Cureus}

impact of coffee and its respective chemical compounds on neurocognitive function and other brain pathologies. It discusses the mechanism of action of its constituents and highlights the degree of importance of coffee according to neurological disorders by comparing them with different studies.

\section{Review}

\section{Bioactive compounds of coffee and their properties}

The most important compounds in coffee include methylxanthines (caffeine, theophylline and theobromine), polyphenols (CGAs and their derivatives like caffeic acid and pyrocatechol), diterpenes comprising cafestol and kahweol, flavonoids (anthocyanin and catechins), lactones, trigonelline, nicotinic acid (vitamin B3), and many more micronutrients like potassium and magnesium [3,6,15]. All of these have many physiological effects, some overlapping with others. When coffee undergoes roasting, amino acids and carbohydrates react through a process called Maillard reactions that produce additional compounds like melanoidins and acrylamide $[4,6]$. Although little is known, caffeine and polyphenols currently have great neuroprotective properties [14].

The widely studied caffeine has antagonistic activity against adenosine receptors in the central nervous system and elsewhere in the body resulting in psychoactive effects [13]. There is also a notable increase in metabolism, diuresis, and blood pressure in an acute setting [16]. Although caffeine dependence may be questionable as it has been mentioned, studies have shown moderate consumption has not increased dopaminergic transmission in nucleus accumbens, which is a characteristic finding for drugs of dependence $[17,18]$. Nucleus accumbens is the site of the brain that involves the "reward circuit" and is an essential component of the mesolimbic pathway. When this site is stimulated, it releases dopamine that is known for rewarding experiences that are implicated in drug addiction. In patients with headaches and migraines, caffeine may also increase the potency of pain medications and is proven therapeutic [13]. It has decreased the overall risk of developing stroke and neurodegenerative diseases [5]. It is also listed amongst WHO's 2019 list of essential medicines for use in apnea [19,20]. Although it covers innumerable benefits, caffeine intake is also associated with adverse effects in higher doses that are related to jitteriness, palpitations, insomnia, and anxiety [13]. This is due to the short-term effects that raise the sympathetic drive in humans, and thus aggravate conditions related to stress and anxiety. In a systematic review by Vilarim et al., an association of caffeine and panic disorder is indicated [21]. Caffeine is also found in other dietary sources, as shown by (Table 1) [22]. A general trend may be noticed in the table that coffee and its subtypes have the most caffeine except energy drinks.

\begin{tabular}{|c|c|}
\hline Sources of caffeine & Mean concentration \\
\hline Espresso & $60 \mathrm{mg} / 30 \mathrm{~mL}$ \\
\hline Decaffeinated coffee & $3 \mathrm{mg} / 125 \mathrm{~mL}$ \\
\hline Filtered coffee & $85 \mathrm{mg} / 125 \mathrm{~mL}$ \\
\hline Instant coffee & $65 \mathrm{mg} / 125 \mathrm{~mL}$ \\
\hline Tea (leaves or bag) & 32 mg/150 mL \\
\hline Iced tea & $20 \mathrm{mg} / 330 \mathrm{~mL}$ \\
\hline Caffeinated soft drinks & $39 \mathrm{mg} / 330 \mathrm{~mL}$ \\
\hline Sugar-free soft drinks & $41 \mathrm{mg} / 330 \mathrm{~mL}$ \\
\hline Energy drinks & $80 \mathrm{mg} / 330 \mathrm{~mL}$ \\
\hline Hot chocolate & $4 \mathrm{mg} / 150 \mathrm{~mL}$ \\
\hline Chocolate bar & $20 \mathrm{mg} / 30 \mathrm{~g}$ \\
\hline Dark chocolate & $60 \mathrm{mg} / 30 \mathrm{~g}$ \\
\hline Milk chocolate & $6 \mathrm{mg} / 30 \mathrm{q}$ \\
\hline
\end{tabular}

TABLE 1: Sources of caffeine in the diet.

Many studies have also shown coffee to attenuate inflammatory chemokines, such as interleukin 6, tumor necrosis factor- $\alpha$, interferon $-\gamma$, and transforming growth factor- $\beta$. The main contributors being diterpenes, caffeine, trigonelline, and CGAs [23]. Caffeine itself demonstrates both anti-inflammatory and proinflammatory activity, as interpreted by Paiva et al. [24]. These benefits might play a crucial part in 
Diterpenes like cafestol and kahweol exhibit important antioxidant and chemoprotective properties but also have raised serum cholesterol levels $[25,26]$. Antioxidant properties of chemical compounds generally eliminate free radicals in the human body. Free radicals, when in excess, cause oxidative stress leading to DNA damage and cell death. This further increases the risk of diseases like cancer. Trigonelline, along with melanoidins, also have antioxidant effects by similar mechanisms [27,28]. CGAs share not only antioxidant characteristics but also anti-inflammatory effects by inhibiting the expression of cytokine genes and modulating inflammatory nuclear factor kappa-light-chain-enhancer of activated B cells (NF-KB) activation and is associated with regeneration of neurons contributing to neuroplasticity $[13,15]$.

\section{Neurocognitive decline, dementia, and AD}

Age-related cognitive decline is a growing issue in the elderly population [29]. Its progression to dementia is a continuous, irreversible process with treatment options that are very limited; therefore, reducing risk factors for the development of cognitive impairment is paramount. Caffeine attributes to most cognitive benefits. There are mixed studies depicting the positive association of decaffeinated coffee compared to caffeinated coffee affecting cognitive performance [30]. A recent publication by Dong et al. concluded from the National Health and Nutrition Examination Survey (NHANES), which was conducted by Centers for Disease Control and Prevention (CDC), that coffee, caffeinated coffee, and caffeine were associated with cognitive performance while decaffeinated coffee was not [30].

Moreover, studies also show this association in a dose-dependent manner, a greater number of coffee cups lower the risk of cognitive impairment, dementia, and $\mathrm{AD}$ [31]. An observational meta-analysis did observe positive effects of coffee (caffeine) on cognitive disorders with a relative risk (RR) of 0.84 (95\% CI: 0.72-0.99, I2 $=42.6 \%$ ) but then again another observational meta-analysis did not find such association [32,33]. The mechanism by which this occurs remains unclear. A study suggested it acts through cholinergic pathways that specifically enhance memory in humans [34]. Various animal studies show how caffeine enhances memory as well $[35,36]$. An overview of studies discussing coffee/caffeine intake with cognitive impairment and their results are shown in Table 2.

\begin{tabular}{|c|c|c|c|c|c|}
\hline $\begin{array}{l}\text { Authors } \\
\text { (reference) }\end{array}$ & $\begin{array}{l}\text { Year of publication } \\
\text { range }\end{array}$ & Type of study & $\begin{array}{l}\text { Number of } \\
\text { participants }\end{array}$ & $\begin{array}{l}\text { Parameter used with } \\
95 \% \mathrm{Cl}\end{array}$ & $\begin{array}{l}\text { Association found } \\
\text { (yes/no) }\end{array}$ \\
\hline $\begin{array}{l}\text { Dong } x \text { et al. } \\
\text { [30] }\end{array}$ & 2011-2014 & Meta-analysıs & 2,513 & OR: 0.56 (0.35-0.89) & Yes \\
\hline Wu L et al. [31] & Inception-2016 & Meta-analysis & 34,282 & RR: 0.82 (0.71-0.94) & Yes \\
\hline $\begin{array}{l}\text { Santos C et al. } \\
\text { [32] }\end{array}$ & $1989-2009$ & $\begin{array}{l}\text { Systematic review/meta- } \\
\text { analysis }\end{array}$ & - & RR: $0.84(0.12-0.99)$ & Yes \\
\hline $\begin{array}{l}\text { KIm YS et al. } \\
\text { [33] }\end{array}$ & Inception-2014 & Meta-analysis & $31,4 / 99$ & $\begin{array}{l}\text { OR/RR: } 0.83(0.70- \\
0.98)\end{array}$ & No \\
\hline $\begin{array}{l}\text { Solfrizzi V et } \\
\text { al. [39] }\end{array}$ & - & Longitudinal study & 1,445 & HR: $0.26(0.03-2.11)$ & no \\
\hline
\end{tabular}

TABLE 2: Articles published demonstrating association of coffee/caffeine consumption with cognitive impairment.

$\mathrm{Cl}$, confidence interval; RR, relative risk; OR, odds ratio; HR, hazard ratio.

$\mathrm{AD}$ is the most common cause of dementia [37]. Several studies have suggested that coffee is associated with reduced risks of developing $\mathrm{AD}[16]$. This could be due to the effects of caffeine and CGAs on the adenosine receptors that ultimately play a role in preventing toxic $\beta$-amyloid peptide deposits in the brain [37]. This mechanism can be very specific to the extent and type of adenosine receptors involved because animal models with $\mathrm{AD}$ observed the neuroprotective potential of caffeine due to its action on A2AR rather than A1 receptors [38]. This indicates that adequate adenosinergic activation is needed for normal memory function and that an over- or underactivation can lead to memory impairment [39]. This further explains why a recent study states coffee intake of $\geqslant 2$ cups/day was significantly associated with a lower $A \beta$ positivity compared to lower coffee drinkers ( $<2$ cups/day), resulting in decreased cerebral amyloid deposition [40]. As the benefits of coffee on these disorders are much attributed to methylxanthines (i.e., caffeine), Figure 1 illustrates how inhibiting the subtypes of adenosine receptors can have different effects on them. Adenosine A1 receptors enhance the release of neurotransmitters, while A2A receptors focus on improving neurocognitive function and neuroplasticity. This can be explored in greater depth as a pharmacological approach for such disease 


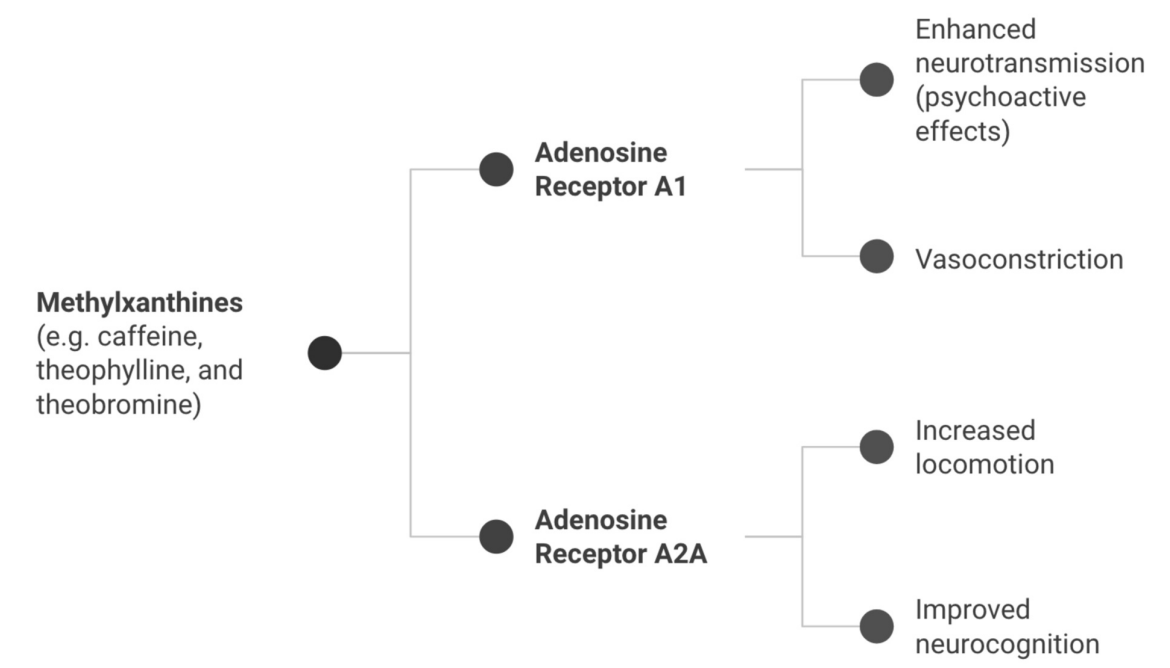

FIGURE 1: Effects of adenosine antagonism by methylxanthines in the brain.

\section{Parkinson's disease}

Many epidemiological studies have suggested an association of coffee with the development of PD, while some may contradict with others $[5,13,16,25,41]$. The neuroprotective properties seen are attributed to caffeine and CGSs [25,37]. Again, there is a dose-relationship pattern that is observed in PD to achieve the most benefit from coffee being about 3 cups/day [42]. Coffee not only reduces the risk of PD but in a prospective study $(\mathrm{n}=16)$ appeared to improve "total akinesia" type of freezing on gait but for a limited number of months after which the patients developed tolerance. A two-week caffeine withdrawal restored these effects [43].

These potential beneficial effects of coffee (caffeine/CGAs) can be due to adenosine receptor antagonism in dopamine-rich areas of the brain that augment its release and enhance transmission [13,25]. A study in mice demonstrated neuroprotection on 1-methyl-4-phenyl-1,2,3,6 tetra-hydropyridine (MPTP) model of PD by virtue of A2AR activity [44]. The local antioxidant property of CGAs aids in neurogenesis and together with pyrogallol, trigonelline, catechol, 5-hydroxytryptamides, and $\mathrm{N}$-methylpyridinium exhibits similar effects by increasing calcium signaling and dopamine release in the central nervous system [13,41]. Another explanation is that coffee raises the number of bifidobacteria that are associated with mitigating local inflammatory response, diminishing procarcinogenic processes and lower misfolding rates of $\alpha$-synuclein in the enteric nervous system, therefore reducing the risk of PD by decreasing dissemination of the protein to the brain [45]. There are very few studies that have looked into this avenue and require further investigation to better understand the mechanism. It is of note that the strongest association among all neurodegenerative diseases has been indicated in caffeine consumption with PD incidence [46].

\section{Stroke}

Several studies mention the association of coffee with the risk of stroke $[5,6,13]$. Patil et al. discusses many epidemiological studies regarding high coffee consumption, one being associated with a lower risk of total stroke, cerebral infarction, and subarachnoid hemorrhage but not intracerebral hemorrhage. This was after other risk factor adjustments were made [16]. A large meta-analysis of 36 cohort studies comprising 36,352 cases of cardiovascular diseases including stroke reported a $5 \%$ decrease in relative risk of stroke by a median consumption of 5 cups daily and $15 \%$ for 3.5 cups compared with a median consumption of zero [47]. Most prospective studies support a weak inverse proportionality between moderate coffee consumption and stroke risk [48].

Further placebo studies are needed to clarify the mechanisms and to elucidate a plausible causal relationship. Lifetime coffee intake may lead to late life cerebral white matter hyperintensities in cognitively normal elderly individuals, which signify cerebral hypoperfusion [49]. This might be attributed to the prolonged exposure of caffeine that results in vasoconstriction and decreases cerebral blood flow over time. It was seen in a Korean longitudinal study with women more affected than men, which can be explained by certain hormones (estradiol) that decrease the clearance of caffeine in women [49]. This gender disparity should also be taken into account when studies are being done. 


\section{Others}

Several studies discuss the implications of coffee in other diseases. Its role in depression has also been quite promising [14,37]. Nehlig mentions the effects of caffeine in elevating mood and reducing depression, contributing to the psychoactive properties of coffee [13]. Many people take coffee to drive sleep away and to prevent the drowsiness associated with the postprandial alkaline tide. A study by George et al. states that there is no clear evidence of caffeine consumption with sleep problems; however, a more recent study found that high lifetime coffee consumption decreases the overall pineal gland volume and this might disturb the quality of sleep later on in life [12,50]. In the management of many cases, coffee is to be avoided in the evening for people with sleep problems.

In a few experimental studies, caffeine showed a positive impact on the course of multiple sclerosis rather than affecting the development of the disease. On the other hand, no such benefits were observed in clinical and experimental studies on amyotrophic lateral sclerosis [50]. Other than this, there are many more benefits of coffee seen that include a better prognosis of patients with traumatic brain injury. The correlation in these patients was with higher cerebral spinal fluid caffeine levels [46].

\section{Conclusions}

Coffee may reduce the overall risk of neurocognitive decline and play a beneficial role in other neurodegenerative diseases when consumed in moderate amounts. It is associated with very few harmful effects only in high doses. The exact mechanisms by which each component affects the brain still need to be further investigated on humans. Each compound and its effects must be studied separately as they have their own unique properties that can have different purposes according to the type of disease.

The vast majority of studies discuss the association of coffee with different health outcomes but no causal relationship is identified. This is primarily due to the presence of observational studies that provide low quality evidence. Therefore we call upon randomized controlled trials to study coffee consumption in a dose-dependent manner within healthy and patient populations. Furthermore, it is essential to remove confounders and take into account the physiologic parameters and genetic variations that could alter conclusions drawn from studies by categorization. This way we may raise the statistical power of studies and better understand the potential pharmacological role of coffee in various disease processes.

\section{Additional Information \\ Disclosures}

Conflicts of interest: In compliance with the ICMJE uniform disclosure form, all authors declare the following: Payment/services info: All authors have declared that no financial support was received from any organization for the submitted work. Financial relationships: All authors have declared that they have no financial relationships at present or within the previous three years with any organizations that might have an interest in the submitted work. Other relationships: All authors have declared that there are no other relationships or activities that could appear to have influenced the submitted work.

\section{References}

1. How many cups of coffee (7 oz), on average, do you drink per day? . (2017). Accessed: July 17, 2020: https://www.statista.com/statistics/695772/average-daily-coffee-consumption-by-country/.

2. What's new. (2020). Accessed: June 2, 2020: http://www.ico.org/.

3. Jeszka-Skowron M, Zgoła-Grześkowiak A, Grześkowiak T: Analytical methods applied for the characterization and the determination of bioactive compounds in coffee. Eur Food Res Technol. 2015, 240:19-31. 10.1007/s00217-014-2356-Z

4. Hu GL, Wang X, Zhang L, Qiu MH: The sources and mechanisms of bioactive ingredients in coffee . Food Funct. 2019, 10:3113-3126. 10.1039/c9fo00288j

5. Poole R, Kennedy OJ, Roderick P, Fallowfield JA, Hayes PC, Parkes J: Coffee consumption and health: umbrella review of meta-analyses of multiple health outcomes. BMJ. 2017, 359:j5024. 10.1136/bmj.j5024

6. Nieber K: The impact of coffee on health. Planta Med. 2017, 83:1256-1263. 10.1055/s-0043-115007

7. Kim Y, Je Y, Giovannucci E: Coffee consumption and all-cause and cause-specific mortality: a meta-analysis by potential modifiers. Eur J Epidemiol. 2019, 34:731-752. 10.1007/s10654-019-00524-3

8. Casal S, Oliveira MB, Alves MR, Ferreira MA: Discriminate analysis of roasted coffee varieties for trigonelline, nicotinic acid, and caffeine content. J Agric Food Chem. 2000, 48:3420-3424. 10.1021/jf990702b

9. Gloess AN, Schönbächler B, Klopprogge B, et al.: Comparison of nine common coffee extraction methods: instrumental and sensory analysis. Eur Food ResTechnol. 2013, 236:607-627. 10.1007/s00217-013-1917-X

10. Parras P, Martínez-Tomé M, Jiménez AM, Murcia MA: Antioxidant capacity of coffees of several origins brewed following three different procedures. Food Chem. 2007, 102:582-592. 10.1016/j.foodchem.2006.05.037

11. Guertin KA, Loftfield E, Boca SM, et al.: Serum biomarkers of habitual coffee consumption may provide insight into the mechanism underlying the association between coffee consumption and colorectal cancer. Am J Clin Nutr. 2015, 101:1000-1011. 10.3945/ajcn.114.096099

12. George SE, Ramalakshmi K, Mohan Rao LJ: A perception on the health benefits of coffee . Crit Rev Food Sci Nutr. 2008, 48:464-486. 10.1080/10408390701522445 
13. Nehlig A: Effects of coffee/caffeine on brain health and disease: What should I tell my patients? . Pract Neurol. 2016, 16:89-95. 10.1136/practneurol-2015-001162

14. Islam MT, Tabrez S, Jabir NR, et al.: An insight into the therapeutic potential of major coffee components . Curr Drug Metab. 2018, 19:544-556. 10.2174/1389200219666180302154551

15. Funakoshi-Tago M, Nonaka Y, Tago K, et al.: Pyrocatechol, a component of coffee, suppresses LPS-induced inflammatory responses by inhibiting NF-KB and activating Nrf2. Sci Rep. 2020, 10:2584. 10.1038/s41598020-59380-x

16. Patil H, Lavie CJ, O’Keefe JH: Cuppa joe: friend or foe? Effects of chronic coffee consumption on cardiovascular and brain health. Mo Med. 2011, 108:431-438.

17. Iancu I, Olmer A, Strous R: Caffeinism: history, clinical features, diagnosis, and treatment . Caffeine and Activation Theory: Effects on Health and Behavior. Smith B, Gupta U, Gupta B (ed): CRC Press, Florida; 2007. 331-344.

18. De Luca MA, Bassareo V, Bauer A, Di Chiara G: Caffeine and accumbens shell dopamine. J Neurochem. 2007, 103:157-163. 10.1111/j.1471-4159.2007.04754.x

19. Executive summary the selection and use of essential medicines 2019 . (2019). Accessed: June 2, 2020: https://apps.who.int/iris/bitstream/handle/10665/325773/WHO-MVP-EMP-IAU-2019.05-eng.pdf?ua=1.

20. Schmidt B, Anderson PJ, Doyle LW, et al.: Survival without disability to age 5 years after neonatal caffeine therapy for apnea of prematurity. JAMA. 2012, 307:275-282. 10.1001/jama.2011.2024

21. Vilarim MM, Rocha Araujo DM, Nardi AE: Caffeine challenge test and panic disorder: a systematic literature review. Expert Rev Neurother. 2011, 11:1185-1195. 10.1586/ern.11.83

22. Sources of caffeine. (2020). Accessed: June 15, 2020: https://www.coffeeandhealth.org/topicoverview/sources-of-caffeine/.

23. Martini D, Del Bo C, Tassotti M, Riso P, Del Rio D, Brighenti F, Porrini M: Coffee consumption and oxidative stress: a review of human intervention studies. Molecules. 2016, 21:979. 10.3390/molecules21080979

24. Paiva C, Beserra B, Reis C, Dorea JG, Da Costa T, Amato AA: Consumption of coffee or caffeine and serum concentration of inflammatory markers: a systematic review. Crit Rev Food Sci Nutr. 2019, 59:652-663. 10.1080/10408398.2017.1386159

25. Gökcen BB, Şanlier N: Coffee consumption and disease correlations . Crit Rev Food Sci Nutr. 2019, 59:336348. 10.1080/10408398.2017.1369391

26. Penson P, Serban MC, Ursoniu S, Banach M: Does coffee consumption alter plasma lipoprotein (A) concentrations? A systematic review. Crit Rev Food Sci Nutr. 2018, 58:1706-1714. 10.1080/10408398.2016.1272045

27. Zhou J, Zhou S, Zeng S: Experimental diabetes treated with trigonelline: effect on $\beta$ cell and pancreatic oxidative parameters. Fundam Clin Pharmacol. 2013, 27 :279-287. 10.1111/j.1472-8206.2011.01022.x

28. Moreira AS, Nunes FM, Domingues MR, Coimbra MA: Coffee melanoidins: structures, mechanisms of formation and potential health impacts. Food Funct. 2012, 3:903-915. 10.1039/c2fo30048f

29. Centers for Disease Control and Prevention. The Healthy Brain Initiative: the public health road map for state and national partnerships, 2013-2018, Interim Progress Report. (2015). Accessed: June 2, 2020: https://www.cdc.gov/aging/pdf/2013-healthy-brain-initiative-interim-report.pdf.

30. Dong X, Li S, Sun J, Li Y, Zhang D: Association of coffee, decaffeinated coffee and caffeine intake from coffee with cognitive performance in older adults: National Health and Nutrition Examination Survey (NHANES) 2011-2014. Nutrients. 2020, 12:840. 10.3390/nu12030840

31. Wu L, Sun D, He Y: Coffee intake and the incident risk of cognitive disorders: a dose-response meta-analysis of nine prospective cohort studies. Clin Nutr. 2017, 36:730-736. 10.1016/j.clnu.2016.05.015

32. Santos C, Costa J, Santos J, Vaz-Carneiro A, Lunet N: Caffeine intake and dementia: systematic review and meta-analysis. J Alzheimer's Dis. 2010, 20:187-204. 10.3233/JAD-2010-091387

33. Kim YS, Kwak SM, Myung SK: Caffeine intake from coffee or tea and cognitive disorders: a meta-analysis of observational studies. Neuroepidemiology. 2015, 44:51-63. 10.1159/000371710

34. Riedel W, Hogervorst E, Leboux R, Verhey F, van Praag H, Jolles J: Caffeine attenuates scopolamine-induced memory impairment in humans. Psychopharmacology. 1995, 122:158-168. 10.1007/BF02246090

35. Onaolapo AY, Onaolapo OJ: Caffeine's influence on object recognition and working-memory in prepubertal mice and its modulation by gender. Pathophysiology. 2015, 22:223-230.

36. Pierard C, Krazem A, Henkous N, Decorte L, Béracochéa D: Acute stress blocks the caffeine-induced enhancement of contextual memory retrieval in mice. Eur J Pharmacol. 2015, 761:70-78. 10.1016/j.ejphar.2015.04.030

37. Farah A, da Paula Lima J: Consumption of chlorogenic acids through coffee and health implications . Beverages. 2019, 5:11. 10.3390/beverages5010011

38. Dall'Igna OP, Fett P, Gomes MW, Souza DO, Cunha RA, Lara DR: Caffeine and adenosine A2A receptor antagonists prevent beta-amyloid (25-35)-induced cognitive deficits in mice. Exp Neurol. 2007, 203:241245. 10.1016/j.expneurol.2006.08.008

39. Solfrizzi V, Panza F, Imbimbo BP, et al.: Coffee consumption habits and the risk of mild cognitive impairment: the Italian longitudinal study on aging. J Alzheimers Dis. 2015, 47:889-899. 10.3233/JAD150333

40. Kim JW, Byun MS, Yi D, et al.: Coffee intake and decreased amyloid pathology in human brain . Transl Psychiatry. 2019, 9:270. 10.1038/s41398-019-0604-5

41. Herden L, Weissert R: The impact of coffee and caffeine on multiple sclerosis compared to other neurodegenerative diseases. Front Nutr. 2018, 5:133. 10.3389/fnut.2018.00133

42. Qi H, Li S: Dose-response meta-analysis on coffee, tea and caffeine consumption with risk of Parkinson's disease. Geriatr Gerontol Int. 2014, 14:430-439. 10.1111/ggi.12123

43. Kitagawa M, Houzen H, Tashiro K: Effects of caffeine on the freezing of gait in Parkinson's disease . Mov Disord. 2007, 22:710-712. 10.1002/mds.21208

44. Xu K, Di Luca DG, Orrú M, Xu Y, Chen JF, Schwarzschild MA: Neuroprotection by caffeine in the MPTP model of Parkinson's disease and its dependence on adenosine A2A receptors. Neuroscience. 2016, 322:129137. 10.1016/j.neuroscience.2016.02.035 


\section{Cureus}

45. Mulak A, Bonaz B: Brain-gut-microbiota axis in Parkinson’s disease. World J Gastroenterol. 2015, 21:1060910620. 10.3748/wjg.v21.i37.10609

46. Camandola S, Plick N, Mattson MP: Impact of coffee and cacao purine metabolites on neuroplasticity and neurodegenerative disease. Neurochem Res. 2019, 44:214-227. 10.1007/s11064-018-2492-0

47. Ding M, Bhupathiraju SN, Satija A, van Dam RM, Hu FB: Long-term coffee consumption and risk of cardiovascular disease: a systematic review and a dose-response meta-analysis of prospective cohort studies. Circulation. 2014, 129:643-659. 10.1161/CIRCULATIONAHA.113.005925

48. Larsson SC: Coffee, tea, and cocoa and risk of stroke. Stroke. 2014, 45:309-314. 10.1161/STROKEAHA.113.003131

49. Park J, Han JW, Lee JR, Byun S, Suh SW, Kim JH, Kim KW : Association between lifetime coffee consumption and late life cerebral white matter hyperintensities in cognitively normal elderly individuals. Sci Rep. 2020, 10:421. 10.1038/s41598-019-57381-z

50. Park J, Han JW, Lee JR, et al.: Lifetime coffee consumption, pineal gland volume, and sleep quality in late life. Sleep. 2018, 41:zsy127. 10.1093/sleep/zsy127 